\title{
ZnO Nano-microstructures and their Photo Conducting Properties Synthesized by Sol-Gel Method
}

\author{
Sheo K. Mishra ${ }^{* 1}$, U.K. Tripathi ${ }^{2}$, Saurabh Dixit ${ }^{3}$, K.C. Dubey ${ }^{4}$ and R.K. Shukla ${ }^{5}$
}

1,2,3. Central Institute of Plastics Engineering and Technology (CIPET), Lucknow-226008, (U.P.), India

4,5. Department of Physics, University of Lucknow, Lucknow-226007, (U.P.), India

\section{Publication Info}

\section{Article history :}

Received : $01^{\text {st }}$ Sept., 2018

Accepted : $17^{\text {th }}$ Nov., 2018

DOI : $10.18090 /$ samriddhi.v10i02.3

Keywords : ZnO, Sol-gel, XRD, Photoconductivity.

*Corresponding author :

Sheo K. Mishra

e-mail : dr.sheokmishra@gmail.com

\begin{abstract}
In the present paper, photoconductivity $(P C)$ of Zinc-oxide $(\mathrm{ZnO})$ nanostructures synthesized by sol-gel method has been investigated. Structural study has been performed by X-ray diffraction (XRD) patterns. The XRD result confirms the formation of hexagonal phase. In the photoconductivity analysis, growth and decay of photocurrent of $\mathrm{ZnO}$ nanostructures have been investigated. The photo-response of prepared sample has been measured under UV illumination using thick film of powder without any binder. The growth and decay of photocurrent show fast rise and decay of photocurrent indicating suitability for UV photodetectors applications.
\end{abstract}

\section{INTRODUCTION}

$\mathrm{D}$ etection of ultra-violet (UV) radiations is the subject of extensive study for the scientific communities and researchers to explore their possible applications for both civilian as well as military areas such as flame sensing, optical and space communication etc. [1]. $\mathrm{ZnO}$ is II-VI semiconductor with a wide band gap of $3.37 \mathrm{eV}$ and a large exciton binding energy of $60 \mathrm{meV}$, making it one of the most attractive materials for potential applications. In addition, $\mathrm{ZnO}$ exhibits an n-type characteristic with a large free electron concentration originating from native defects such as oxygen vacancies and zinc interstitials [2, 3]. Various methods have been reported for preparing $\mathrm{ZnO}$ nanostructures including thermal decomposition method [2, 4], solid state reaction method [5], precipitation method [6], hydrothermal method [3] and sol-gel method [7]. Among these synthesis methods, sol-gel method has attracted considerable attention because of its flexibility in fine tailoring of nanoparticles properties.

Light emission and detection uniqueness of $\mathrm{ZnO}$ nanostructures in ultraviolet (UV) region are attractive aspects for the fabrication of UV light emitting diodes and UV photodetectors [8, 9]. With the rapid development in experimental facilities and sophisticated techniques, it has been possible to fabricate quality UV photodetectors based on $\mathrm{ZnO}$ nanostructure [10, 11]. UV photodetector based on colloidal $\mathrm{ZnO}$ nanoparticles has been found to exhibit high efficiency [10]. In this paper, we have studied photoconductivity of $\mathrm{ZnO}$ nanostructures synthesized by sol-gel method.

\section{EXPERIMENTAL SECTION}

\subsection{Sample Preparation}

The $\mathrm{ZnO}$ nanostructures were synthesized using $\mathrm{Zn}\left(\mathrm{CH}_{3} \mathrm{COO}\right)_{2} \cdot\left(2 \mathrm{H}_{2} \mathrm{O}\right)$, Iso-propanol, and Diethyl Amine using the surfactant assisted complex sol-gel method [12]. The mixed transparent 
solution containing $0.1 \mathrm{~mol} \mathrm{Zn}\left(\mathrm{CH}_{3} \mathrm{COO}\right)_{2} \cdot\left(2 \mathrm{H}_{2} \mathrm{O}\right)$ was dissolved in $50 \mathrm{ml}$ Isopropenal with few drops of Dimethyl Amine. The solution was stirred for 48 to $72 \mathrm{~h}$ at room temperature. The transparent solution was gelled and was immediately put into a furnace at $373 \mathrm{~K}$ for $12 \mathrm{~h}$ in air. The nanopowders were milled in an agate mortar.

\subsection{Characterizations}

The structural analysis of $\mathrm{ZnO}$ NPs have been done by X-ray diffraction (XRD) using Rigaku D/ MAX- 2200H/PC, $\mathrm{Cu} \mathrm{K \alpha}(\sim 1.5404 \AA)$ radiation. For photoconductivity measurements, a cell type device is used. The cell is formed by putting a thick layer of powdered sample in between two $\mathrm{Cu}$ electrodes having a spacing of $1 \mathrm{~mm}$. The powdered layer is pressed with a glass plate for providing illumination area of $0.25 \mathrm{~cm}^{2}$. The cell is mounted in a dark chamber. The light illumination is allowed to fall over the cell with a slit. The Hg lamp of 300 $\mathrm{W}$ is used as photo-excitation source. The cell is first kept in the dark for several hours. The temporal response i.e. growth and decay of photocurrent are recorded using RISH Multi $15 \mathrm{~S}$ with adapter RISH Multi SI-232. All of the experiments are done at room temperature in ambient air.

\section{RESULTS AND DISCUSSION}

\subsection{X-ray Diffraction (XRD)}

Fig. 1 shows XRD pattern of ZnO nanostructures synthesized by sol-gel method. The XRD patterns are broadened due to small size of crystallites, which is the confirmation of nano-sized particles formation. The presence of various diffraction peaks suggests polycrystalline nature and indicates formation of hexagonal wurtzite phase of $\mathrm{ZnO}$. The XRD pattern exhibits no additional peak due to by-products such as $\mathrm{Zn}(\mathrm{OH})_{2}$. The crystallite size of $\mathrm{ZnO}$ sample is calculated using Scherrer's formula [2, 3]: $D=0.91 \lambda / \beta \cos \theta$, where $\lambda$ is the wavelength of $\mathrm{X}$-ray radiation, $\beta$ is the full width at half maximum (FWHM) of the peaks at the diffracting angle $\theta$. The estimated average crystallite size of $\mathrm{ZnO}$ NPs corresponding to the maximum intensity peaks is to be $\sim 38.22 \mathrm{~nm}$.

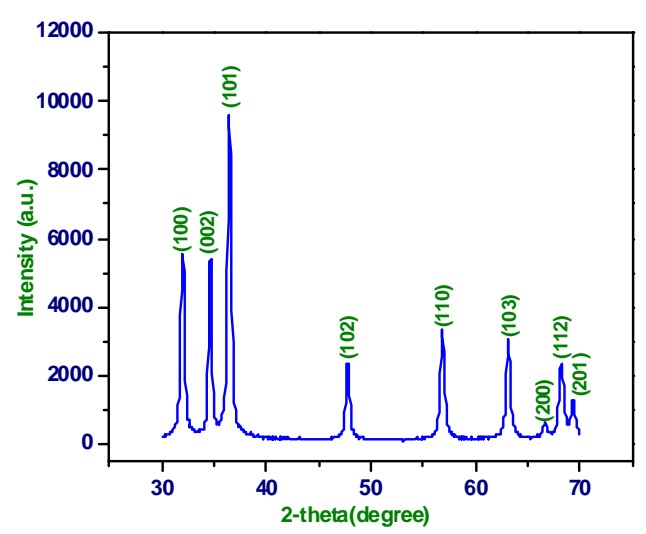

Fig.1: X-ray diffraction pattern of $\mathrm{ZnO}$ NPs with average crystallite size of $\sim 38.22 \mathrm{~nm}$.

\subsection{Growth and Decay of Photocurrent}

Photoconductivity in $\mathrm{ZnO}$ consists of two processes; one is a bulk-related process, which is a fast process associated with the oxygen molecules adsorbed in the grain boundaries of the material. On the other hand, it is a surface-related process, which is a slow process which is attributed to the processes of adsorption and photodesorption of $\mathrm{O}_{2}$ molecules on the surface of nanostructures. In photoconductivity study, the variation of photocurrent as a function of various parameters including applied voltage, energy of illumination, temperature, and other parameters gives us very efficient information regarding the nanomaterials [2,3]. In the present work, temporal response i.e. growth and decay of photocurrent of $\mathrm{ZnO}$ nanostructures prepared by sol-gel method has been investigated which is shown in Fig. 2 . Initially, when the field is applied across the cell the dark-current is found which starts decreasing until it gets nearly saturated. At this moment light is switched-On and the photocurrent increases and takes $\sim 12.9$ minutes to attain its maximum. When the light is switched-Off, the photocurrent 
decreases rapidly and it reaches $80 \%$ of photocurrent within $\sim 4$ mints.

It is well known that at equilibrium in the dark, oxygen molecules of the atmosphere are adsorbed onto the $\mathrm{ZnO}$ nanorod surfaces by capturing the free electrons from the n-type $\mathrm{ZnO}$ due to oxygen vacancy as follows: $\mathrm{O} 2(\mathrm{~g})+\mathrm{e}^{-} \rightarrow \mathrm{O}^{-2}(\mathrm{ad})$. These adsorbed oxygen ions create a low-conductivity depletion layer near $\mathrm{ZnO}$ surfaces [1-3, 13-14]. These adsorbed atoms decrease the conductivity by a reduction of both the density of free electrons and effective conducting thickness of the nanorods. Due to large surface-to-volume ratio of nanostructures, these effects are more prominent and play an important role in photo-detection of materials. On illumination of UV light of photon energies above the band gap, electron-hole pairs (EHPs) are generated. The photo-generated holes migrate to surface along the potential gradient produced by band bending either via discharge of the negatively charged adsorbed $\mathrm{O}_{2}$ ions through $\mathrm{h}^{+}+\mathrm{O}^{-2}(\mathrm{ad}) \rightarrow \mathrm{O}_{2}(\mathrm{~g})$ to photodesorb oxygen from the surfaces or effectively get trapped at the surfaces, resulting in an increase in the free carrier concentration and a decrease in the width of depletion layer [2, 3]. This leads to an enhancement of carrier injection and transport, producing a persistent photocurrent. When the illumination is switched-Off, oxygen readsorbs on the surfaces of nanostructures, and the photocurrent decreases.

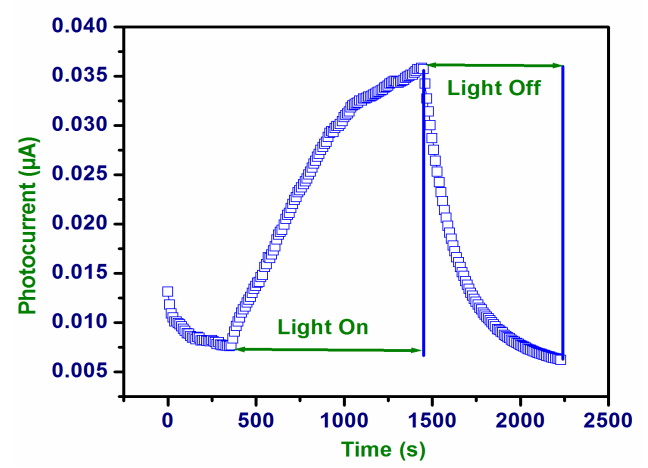

Fig.2: Growth and decay of photocurrent with time for ZnO NPs synthesized by sol-gel method (at V $=10 \mathrm{~V}$ ).

\section{CONCLUSION}

In conclusion, we have synthesized $\mathrm{ZnO}$ nanostructures by sol-gel method. The XRD patterns are broadened due to small size of crystallites, which is the confirmation of nanosized particles formation with average crystallite size $\sim 38.22 \mathrm{~nm}$ corresponding most intense peak. The growth and decay curve shows fast and slow components which is investigated. Further results are still under investigations.

\section{REFERENCES}

[1] Y. Jin, J. Wang, B. Sun, J. C. Blakesley and N.C. Greenham, Nano Lett. 8 1649-1653 (2008).

[2] S. K. Mishra, R. K. Srivastava, S. G. Prakash, J. Alloys Comp. 539, 1-6 (2012).

[3] S. K. Mishra, R. K. Srivastava, S. G. Prakash, R. S. Yadav and A. C. Pandey, Opto- Electron. Rev. 18, 467 (2010).

[4] S. K. Mishra, S. Bayan, P. Chakraborty, R. K. Srivastava, Applied Physics A: Materials Science \& Processing 115, 1193-1203 (2014)

[5] S. K. Mishra, R. K. Srivastava, S. G. Prakash, J Mater Sci: Mater Electron 24, 125-134 (2013)

[6] R. Kripal, A. K. Gupta, R. K. Srivastava and S. K. Mishra, Spectrochimica Acta Part A 79, 1605 (2011).

[7] S. K. Mishra, S. Bayan, R. Shanker, P. Chakraborty, R. K. Srivastava, Sensors Actuators A 211, 8-14 (2014).

[8] J. Wang and L. Gao, J. Mater. Chem. 13, 2551 (2003)

[9] C.Y. Liu, B.P. Zhang, Z.W. Lu, N.T. Binh, K. Wakatsuki, Y. Segawa and R. Mu, J. Mater. Sci. Mater. Electron. 20, 197 (2009)

[10] Y. Jin, J. Wang, B. Sun, J. C. Blakesley and N.C. Greenham, Nano Lett. 8, 1649 (2008)

[11] Y. Li, F.D. Valle, M. Simonnet, I. Yamada and J.-J. Delaunay, Nanotechnology 20, 045501 (2009)

[12] Y. Zhang, Y. Yang, J. Zhao, R. Tan, W. Wang, P. Cui, W. Song, J Mater Sci 46, 774-780 (2011).

[13] A. Bera, D. Basak, Appl. Phys. Lett. 94, 163119 (2009).

[14] A. Bera, D. Basak, Appl. Phys. Lett. 93, 053102 (2008) 
